\title{
XIIl.
}

\section{Das Substanzproblem, eine philosophiegeschichtliche Darstellung.}

\author{
Von \\ Dr.-phil. Luise Krieg.
}

Der Glaube an die lebensfrohe Götterwelt des Homer und Hesiod war erschüttert. Mochte der künstlerische Sinn der Griechen sich auch durch, den olympischen Kult befriedigt gefühlt haben, dem denkenden, forschenden Geiste konnte er auf die Dauer nicht genügen. Mit der Sehnsucht nach dem unbekannten Gotte verstärkte sich der Trieb nach Wahrheit. Wer ist der Ursprung alles Seins, wenn es die Olympier nicht sind? Was ist Wahrheit? Was ist das Beharrende, Beständige, Absolute, welches ist die Lebensquelle, die nie versiegt, sondern ewig ist, ohne Anfang, ohne Ende? Man nannte diesen Lebensspender, diesen Grund des Seins die Substanz. Die Griechen suchten sie bald in der stofflichen, bald in der gedanklichen Welt, immer aber war es die Frage: Was ist die wahre ovoía, was ist die Substanz?, die sie bewegte.

War die überlebte Volksreligion eine Vergötterung der Naturkräfte gewesen, so ließ 'man nun den Mythos bei seite und suchte das Wesen der Natur mit dem Verstande zu erfassen. Man wollte den Schleier, mit dem die Außenwelt die Substanz verhüllte, lüften.

Die ersten, die das unternahmen, waren die Milesier. Sie glaubten, die Substanz in einem Urstoff, einem Element gefunden zu haben, aus dem sich alles andere Sein entwickelt. So führte Thales alles Leben auf das Wasser zurück, Anaximander sah die Substanz in einem gedachten Urstoffe, dem $\stackrel{\alpha}{\pi} \varepsilon \varepsilon \iota \rho \circ$, einem unbestimmten, unendlichen Etwas, aus dem sich auf mechanischem Wege durch Aussonderung das Kalte und das Warme, das Flüssige und das Trockene bildete, und Anaximenes erklärte die Luft für den Ursprung alles Seins nach Analogie der menschlichen Seele. Denn wie diese den Körper, so halte die Luft die Welt zusammen. 
Jis war die Liebe zur Natur, die den Milefiern diesen Weg der Forschung wies, während die Pythagoreer, vom Geiste der Musik grtricben, die Lösung des Welträtsels mit Hilfe der Mathematik versuchten. Diese Wissenschaft lehrte sie, daß nur die Zahlen und ihre Verlältnisse unbedingt gewiß, „ohne jeden Lug" sind. Ein Hauch der Ewigkeit wehte sie an bei der Erkenntnis, daß die Eins mit ihrer unendlichen Vermehrbarkeit nach oben und ihrer unendlichen Teilbarkeit nach unten auch das einzige Unendliche, Ewige auf Erden ist. Sie spürten den wahren, ewigen Formen, dem Maß und der Harmonie in der Welt nach, und da sie die mathematische Gesetzlichkeit in der ganzen Natur wiederfanden, so war für sie die Zahl - ein Gedankending - das Element aller Dinge, der Erzeuger alles Lebens. Aus der Eins entwickelten sie den Gegensatz der ungeraden und geraden Zahlen, des Begrenzenden und Unbegrenzten und führten den Begriff des Gegensatzes iiberhaupt in ihrer Welterklärung durch.

Diesem Dualismus traten die Eleaten entgegen. Xenophanes, hingerissen von Bewunderung für die Natur und das Göttliche in ihr, brach in das anbetende Bekenntnis aus: "Gott ist "ैv «ai $\pi \tilde{\alpha} v "$ ". Es ist dasselbe Gefühl, das Kant zu dem Geständnis trieb: „Zwei Dinge erfüllen das Gemüt mit immer neuer und zunehmender Bewunderung und Ehrfurcht, je tiefer und anhaltender sich das Nachdenken damit beschäftigt. Der bestirnte Himmel über mir und das moralische Gesetz in mir." Es ist dasselbe Gefühl, das dem Manschen sicher sagt, das Gemeinsame des Einen und des Alls, des Geistes und der Natur ist das Gättliche, in ihm ist alles. Die Gottheit, das Sein ist nur eines. Denn nur, was widerspruchslos gedacht werden kann, ist wahr, existiert. Die unendliche Zeit, der unendliche Raum existieren. Denn sie haben keinen Gegensatz. Und so dachten sich die Eleaten das wahre Sein unendlich, unteilbar und unkörperlich (Melissos), überall sich selbst gleich; ein Sein, das nur gedacht werden konnte, das aber nicht erkennbar war. Ihre Einheitslehre gipfelte in dem Satze des Parmcnides: „Dasselbe ist Denken und Sein." Die Vielheit der Außenweltdinge und ihre Abwandlungen war für sie nur eine trügerische Vorspiegelung der Sinne.

Gerade diese Veränderlichkeit hielt Heraklit von Ephesos für das Einzige, was immer war und ist und sein wird. Für ihn gab es kein Sein, sondern nur ein Werden, für ihn war das einzig Feststehende 
die Erkenntnis, daß ,alles fließt", daß alles in ewigem Wechsel begriffen ist. Denn erwiesenermaßen erzeugt jedes Ding sein Gegenteil, aber beide sind nur verschiedene Äußerungen ein und derselben Grundkraft, des Feuers, das den ewigen Kreislauf der Dinge hervorruft. Aus dem Feuer wird Wasser, aus diesem Erde, das Trockene verwandelt sich wieder in das Flüssige und dieses in Äther, den feurigen Hauch. So ist das Feuer der Ausgangspunkt der ewigen Ordnung, das Gesetz, das alles Werden, das alle Veränderung bewirkt. Weder die Eleaten mit ihrer starken Einheitslehre vom Sein noch Heraklit mit seiner Lehre vom Werden haben die Welt des Seins und die der Erscheinung in Einklang miteinander gebracht.

Diesen Mangel suchten Empedokles, Anaxagoras und Demokrit zu beseitigen, indem sie zu dem einen unwandelbaren Sein die Bewegung hinzufügten, durch die das Werden erklärt wird. Ihre Substanz besteht aus immer sich gleichbleibenden, unveränderlichen Massenteilchen, die sich nur in verschiedener Menge verbinden und trennen und dadurch die verschiedenen Gegenstände und den Wechsel der Erscheinungen schaffen. Empedokles setzt die Substanz aus

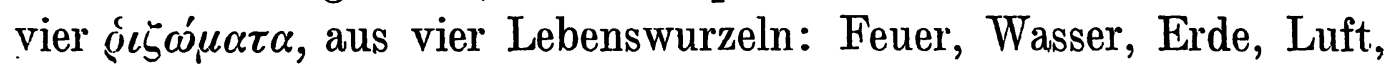
zusammen, die zu einer Kugel vereinigt waren und durch den von außen herantretenden Streit in Bewegung gebracht und voneinander getrennt wurden, so daß sie sich nun verschieden mischen zu organischer und anorganischer Welt. Der Streit hat weiter keine Funktion als die des ersten Bewegers. Die Verbindung der Teile ist daher eine zufällige, aber nur die sinnvollen Körper haben Lebenskraft. Wie bei Empedokles aus Würzelchen, so geht bei Anaxagoras die Welt aus unendlich vielen $\sigma \pi \varepsilon \dot{\varepsilon} \mu \alpha \tau \alpha$ oder Lebenssamen hervor. Sie wurden, damit sie sich verbinden und trennen konnten, von außen her durch den voṽs, die Weltvernunft, bewegt. Dieser voṽs ist die Ursubstanz, das feinste, reinste und leichteste aller Dinge, also noch stofflich gedacht, aber ungemischt, wenn auch teilbar; denn er ist nicht nur der Anstoß zur Bewegung, wir finden ihn auch in den Menschen, den Tieren, den Pflanzen. Im Gegensatz zu dem Streit des Empedokles verfolgt er noch einen besonderen Zweck, die Weltregierung; denn er herrscht mit Einsicht über die Dinge. So unterscheidet Anaxagoras zwei qualitativ voneinander verschiedene Substanzen, den voí

Durch das Moment der Bewegung wird der Gedanke des mecha- 
nischen in die Philosophic eingeführt. Der bedeutendste Vorläufer der modernen mechanischen Naturauffassung ist Demokrit. Die Welt des Seienden, die Substan\%, besteht nach ihm aus unendlich vielen, kleinsten Teilchen, sie sind unteilbar, ungeworden, unvergänglich, unsichtbar, aber doch körperlich gedacht. Ihrer Unteilbarkeit wegen nannte er sie Atome. Sie bewegen sich aus Notwendigkeit, ohne Zweck, und damit sie sich bewegen können, denkt er sie getrennt durch den leeren Raum. Mit dem Begriff der unsichtbaren Atome und des leeren Raumes konstruiert er ein "Sein ohne Materie“. Die Atome sind nur quantitativ voneinander verschieden, nur verschieden an Gestalt, Lage und Größe, „an sich" existiert nur das Atom, die reine Form, die $\sigma \chi \eta_{\eta}^{\prime} \mu \alpha \tau \alpha$. So stellt Demokrit der Stoffsubstanz eines Tales die Formsubstanz gegenüber. Zwischen beiden liegen die mannigfaltigsten Denkmöglichkeiten für Substanzerklärungen. Eine jede könnte, weil logisch folgerichtig aufgebaut, wahr sein. Welche von ihnen entspricht nun der tatsächlichen Welt? Welches ist das Kriterium der Wahrheit?

Gibt es überhaupt eine allgemein gültige Wahrheit? Die Sophisten waren es, die diese Frage aufwarfen und dahin beantworteten, daß für jeden Menschen das wahr sei, was ihm als wahr erscheine. Es gab für sie keine absolute, nur eine relative Wahrheit, eine Erkenntnis, die ihrer Art nach dazu angetan war, zu leugnen und einzureißen. Es mußten erst die ganz Großen kommen, um wieder aufzubauen, die ganz Großen, die den genialen Glauben an eine allgemeingültige Wahrheit in sich trugen und die Kraft spürten, sie zu finden.

Sokrates sah von dem eigentlichen Substanzproblem ab. Ihm lag vielmehr daran zu ergründen, was jeder einzelne Gegenstand ,an sich" sei. Durch die Fragestellung: quid iuris? suchte er die Berechtigung der Benennungen, suchte er das Wesen der einzelnen Dinge aufzudecken und in einer Definition festzuhalten. Durch diese seine Methode wies er seinen größeren Schüler Plato auf das begriffliche Sein hin.

Plato ging den Definitionen auf den Grund und fand, daß die Begriffsbestimmungen uns nur möglich sind, weil wir in unserem Geiste die Idee des wahren Seins haben. Die irdischen Gegenstände sind nur Spezialfälle der Uridee, diese ist das Musterbild, das Allgemeine, von dem die Einzeldinge nur Abbilder sind: Die Vernunftdinge, die Gedankendinge sind das ursprüngliche, das wahrhaft Seiende, 
sie sind die Substanz. Aber wie kamen diese Vernunftdinge in unsere Seele? Die Antwort ist von hinreißender, poetischer Schönheit. Sie sind nicht durch Abstraktion, durch Denkprozesse erworben oder der Seele eingeboren, sondern sie sind Erinnerungen, die die Seele aus ihrem vorirdischen Dasein mit auf diese Welt brachte. Anfangs existierten nur der Demiurg, das formlose Substrat - beide vergänglich gedacht - und die sich selbst stets gleichbleibende Ideenwelt. Aus diesen beiden mischte der Demiurg die Weltseele, aus ihr entstanden die Seelen. Ehe nun die Seelen auf die Erde gingen, traten sie eine Fahrt nach den seligen Gefilden der Wahrheit an, wohnten bei den göttlichen Ideen und brachten von dort Erinnerungen mit, die auf der Erde durch die Sinne getrübt werden. Mag Plato nun den Ideen außerhalb der irdischen Welt eine reale Wirklichkeit zuerkannt haben oder nicht, jedenfalls erklärte er die allgemeine Idee, das Vernunftding, wie wir es in unserem Geiste erschauen, für die Substanz, für das wahre Sein.

An diesem Punkte setzte sein Schüler Aristoteles, der dem hohen Gedankenfluge des Meisters nicht folgen konnte, ein. Ihm ist nicht das Vernunftding die Substanz, für ihn existiert wahrhaft nur der einzelne Gegenstand. Das den einzelnen Gegenständen Gemeinsame - die platonische Idee -, das in sie hineingelegt wird, ist die Substanz. Sie ist der Träger der Eigenschaften, die wir durch die Sinne wahrnehmen. Jeder einzelne Gegenstand hat auch eine Ursache und einen Zweck, die in ihm verborgen liegen und sein Wesen ausmachen. Dies ist der Dualismus von Ursache und Zweck, so daß wir bei Aristoteles schließlich drei Substanzen unterscheiden müssen. Neben dem einzelnen Gegenstande nennt er auch noch Stoff und Form, die Ursache und Zweck entsprechen, als Substanzen. Der Stoff ist gestaltlos und enthält nur die Möglichkeit zu einer realen Existenz in sich. Zur Wirklichkeit erhebt ihn erst die Form, erst sie gibt dem Stoff das Leben, erst sie verleiht ihm das wirkliche Dasein.

Demgegenüber faßten die Stoïker Stoff und Form, Körper und - Geist als eine Einheit auf, für sie war nur der Körper wirklich. Dadurch wurden sie zur Annahme einer stofflich körperlichen Substanz geführt. Alles, was Kraft, was Leben hat, alles, was wirkt, ist Körper, also auch Abstraktes, auch die Seele ist Körper, ist Substanz. Man fing eben an, nach dem thohen Geistesflug der drei Heroen die wahre 
ousìa wicder weniger in der begrifflichen und wieder mehr in der sinnlichen Welt \%u suchen.

Das tat in konsequenter Durchführung Epikur von Samos. Lir wollte die Vielheit der irdischen Erscheinungen dadurch erklären, $\mathrm{da} \beta$ er alles Bestehende in Atome zerlegte. Die Atome sind die Substanz, sie bewegen sich im leeren Raume. Während sie aber bei Demokrit nur gedachte Wirklichkeit besaßen, legte ihnen Epikur reale Wirklichkeit bei unter Leugnung jedes Zweckgedankens.

So lösten die philosophischen Systeme einander ab. Das eine hob das andere auf, und jedes beanspruchte doch Allgemeingültigkeit für sich. Da war es kein Wunder, daß sich schließlich eine skeptische Grundstimmung der Menschheit bemächtigte, die daran verzweifelte, die Beschaffenheit der Dinge „an sich" überhaupt je erfassen zu können. Sie ist unbekannt und wird immer unbekannt bleiben. Denn die Sinne trüben die Kräfte des Verstandes.

Unser Gang durch die griechische Philosophie von Thales zur Skepsis zeigt uns, daß im Altertum der. Begriff der Substanz direkt auf die Dinge selbst geht. Die Substanz ist den Alten eine Form des Seins und darum auch des Denkens. Das Substanzproblem ist hier rein ontologischer Natur.

Im Mittelalter verband sich in der heidnischen wie in der christlichen Philosophie mit dem Wunsche nach Erklärung des rätselvollen Universums eine starke religiöse Sehnsucht und der Glaube an die Unsterblichkeit. Neuplatonismus einerseits, Patristik und Scholastik andrerseits befehdeten und befruchteten einander gleicherweise.

Ihnen allen gemeinsam ist der Gedanke von der Einheit der Substanz, den Plotin als Panentheïsmus entwickelte. Das wahrhafte Sein ist nach ihm das göttliche Ureine, das Ungewordene, die Substanz ohne alle besonderen Eigenschaften, das Absolute schlechthin. Aus ihm geht durch Ausstrahlung die Weltvernunft mit der Ideenwelt hervor, aus dieser die Weltseele, welche die Ideen in der sinnlichen Materie sichtbar darstellt. Die Materie, die Körper sind das gewordene Sein oder nach Plato das Nichtseiende und dementsprechend dàs, was vergeht.

Die chistliche Philosophie führte den Gegenstaz von ungewordenem und gewordenem Sein in das ethische Gebiet über und leitete die Lehre von der Unsterblichkeit der Seele daraus ab. Das Ungewordene 
Das Substanzproblem, eine philosophiegeschichtliche Darstellung. 407

ist das Prinzip des Guten im Menschen, repräsentiert durch die Seele, die, weil ungeworden, nicht vergehen kann, also unsterblich ist. Das Gewordene ist das Prịnzip des Bösen im Menschen, der Körper, der, wie alles gewordene Sein, auch vergänglich ist.

Hatte der Neuplatonismus die Substanz als Einheit aufgefaßt, deren Ausstrahlung das Nichtseiende ist, so sah die christliche Philosophie von der Ausstrahlungstheorie ab und lehrte eine einheitliche göttliche Substanz, die die Fülle der untergeordneten Substanzen umfaßt. Man glaubt an Gott als den Quell alles Seins, aber man identifiziert ihn in keiner Weise mit dem All. Die göttliche Substanz ist die überweltliche Ursache alles Seins.

Dieses Dogma soll nun philosophisch begründet werden, daher die Versuche der Gottesbeweise, deren berühmtester der Anselmsche ist. Anselm konstruiert aus dem reinen Begriff Gottes dessen Existenz. Weil wir uns Gott als schlechthin vollkommen denken, so kann er nicht nur ein Begriff sein. Denn sonst müßten wir uns ein noch vollkommeneres Wesen vorstellen, das auch Realität besäße, also kommt Gott als dem vollkommensten Wesen nicht nur begriffliche, sondern auch objektive Realität zu.

Wie stellte sich nun die christliche Philosophie zu den der göttlichen Substanz untergeordneten Substanzen? Die untergeordneten Substanzen waren die Begriffe und die Körper. Kam den Begriffen oder den Körpern Realität zu, und wie war diese zu denken? Um diese Frage drehte sich der Universalienstreit, der die Gemütër das ganze Mittelalter hindureh erhitzte. Es ist dieselbe Frage, die schon Plato und Aristoteles in Gegensatz zueinander gebracht hatte, die dann die Syrer, später die Araber übernahmen und die nun auf syrischarabischem Wege an die christliche Philosophie des Abendlandes herangebracht wurde. Sind die Vernunftdinge oder die einzelnen Gegenstände das Reale? Im Mittelalter spielt das Moment des zeitlichen prius eine große Rolle, das Ursprüngliche, das Erste ist auch das Reale.

Die Realisten, an ihrer Spitze Scotus Eriugena, erklärten: außerhalb Gottes ist nichts. In ihm sind die Ideen, die Allgemeinbegriffe. Diese sind die Substanzen, welche die einzelnen Gegenstände aus sich heraus entwickeln, bilden. Sie sind daher nicht nur die logischen, sondern die wahrhaft realen Formen des Seins. 
Diesem schroffen Realismus gegenüber nahm Abälard einen gemailigigteren Standpunkt ein. Fr spricht dem Allgemeinen an sich und im besonderen Existenz zu. Die Allgemeinbegriffe, die Ideen, waren in Gott vor aller Zeit, ,vor" allen Dingen. Gott brachte seine Ideen an den einzelnen Gegenständen zur Erscheinung, sie existieren also ,in "den Dingen, sie machen deren eigentliches Wesen aus, und wir finden durch den Denkprozeß der Vergleichung, durch Ausscheidung des bloß Zufälligen und Zusammenfassung des Gemeinsamen, Gleichartigen das Allgemeine, wir schafften es im menschlichen Verstande „nach“ den Dingen. Die Gattungsbegriffe haben demnach ihre Wirklichkeit an den einzelnen Gegenständen.

Den gemäßigten Standpunkt nahm auch Thomas von Aquin ein. Er beweist die Existenz Gottes mit Hilfe des Kausalgesetzes, indem er von der Wirkung, nämlich der Welt, auf die erste Ursache, auf Gott schließt. Nur Gott, die absolute Substanz, existiert an sich, von sich, aus sich selbst. Seine Wirkung, die Welt, stellt ein Stufenreich der substanzialen Formen dar, eine Entwickelungsreihe von den niedrigsten Formen bis hinauf zur Vernunftseele des Menschen, die Gott, das unendliche Sein, erkennen, erfassen möchte.

Im Gegensatz zu den Realisten standen die Nominalisten. Sie hielten die einzelnen Gegenstände für das Ürsprüngliche, für das wirklich Existierende, die Gattungsbegriffe aber nur für Abstraktionen des Verstandes aus den Einzeldingen, also erst nachträglich auf logischem Wege gefunden. Wie damals alle philosophischen Lehren, so wurde auch diese auf das kirchliche Dogma angewandt. Roscelinus erläuterte daran die Unmöglichkeit der göttlichen Trinität, und so wurde der Nominalismus von der Kirche verdammt.

Erst im 14. Jahrhundert gewann er wieder an Bedeutung. Duns Scotus und besonders Occam griffen diese Lehre von neuem auf. Sie schrieben dem Einzeldinge den höheren Wert zu. Denn erst durch die persönliche Note erhalte das Allgemeine das Leben. Die Allgemeinbegriffe seien nur Zeichen, nur termini, die nur das gedachte, das objektive Sein der Dinge angeben. Die einzelnen Gegenstände aber seien das Wirkliche, sie zeigen das Leben, das ,an sich" Sein der Dinge, das subjektive Sein an.

Beide Strömungen, Realismus und Nominalismus enthielten die Keime zu den großen Systemen, die aufzubauen der Neuzeit vorbehalten blieb. Hatte der Realismus das Vernunftding für das einzig 
Reale erkannt, so erklärten nun die neuzeitlichen Rationalisten die Vernunft selbst für das einzige Erkenntnismittel und demzufolge nur das für wahr, was unsere Vernunft erkennt. Aus dem Standpunkt des Nominalismus, der nur die einzelnen Gegenstände als wirklich bezeichnete, ergab sich in konsequenter Weiterführung das Studium der Einzeldinge und nur, was wir von diesen erfahren, ist wahr, lehrten die Empiristen. Die Neuzeit geht also von der Methode aus an alle Probleme und so auch an das Substanzproblem heran.

Die scholastische Philosophie hatte um 1500 abgewirtschaftet, sie stand doch immer mehr: oder weniger unter der Herrschaft des Glaubens. Sie war im ganzen doch die Magd der christlichen Theologie.

Am Eingang zur Neuzeit, ehe wir zu den großen Systemen der Rationalisten und Empiristen gelangen, steht die Renaissance.

Die neue Zeit kündigt sich bereits durch eine ganz andere Fragestellung an. Die Frage: Was ist die Substanz? Was ist die Ursache des Seins, des Lebens? war bis zur Erschöpfung behandelt worden, ohne doch zu einer allgemeingültigen Lösung geführt $\mathrm{zu}$ haben. An Stelle des "Was" interessierte jetzt das "Wie."? Wie wirkt, wie ist die Substanz? Statt der früheren Ansicht, die in den gestaltlosen Stoff die Form von außen hereintrug, vertrat man jetzt den Standpunkt, daß die Substanz durch die in ihr wohnende Kraft wirke. Stoff und Kraft fallen jetzt in eins zusammen, der Sitz des Lebens ist im Stoff selbst. Die poetischen Gemüter faßten die Kraft als Lebensprinzip, als Seele, Weltseele auf und erkannten daher das ganze Weltall als beseelt, während die ernsteren. Naturforscher die Gesetze der Kraft suchten.

Der schönheitstrunkene Panentheïsmus eines Bruno reißt uns noch heute zum heroischen Affekt hin. Gott ist ihm das All und das Eine, die Monade der Monaden, die Substanz. Er baut das ganze Weltall aus unzähligen, graduell verschiedenen Monaden auf, von denen jede eine Offenbarungsform des unendlichen, göttlichen Seins ist. Außerhalb der Monaden gibt es keinen Gott, und Gott ist die Monade. $\mathrm{Zu}$ dieser Erkenntnis führte ihn die Vielheit der Erscheinungen, der Sinneseindrücke, die uns „zwar nicht belügen, uns aber doch nicht die volle Wahrheit sagen". Um diese zu finden, führte er die Vielheit auf die Einheit, auf die Monade zurück. Leider aber 
blieh ar bei dieser mehr intuitiv erschauten Erkenntnis stehen, er unterlie $\mathfrak{B}$ es, die Monade mathematisch zu bestimmen.

Diesen Schritt tat erst Galilei. Als die realen Eigenschaften der J)inge sah er Gestalt, Zahl und Bewegung an. Diese machen die Substanz, das Bestehende im Körper aus, so daß man Gestalt und Zahl als extensive Größen, also als Stoff, Bewegung als intensive Größe, also als Kraft auffassen kann. Weil er durch die Mathematik erkamnt hatte, nur was meßbar ist, ist wahr, so maß er die Größe der Bewegung und stellte ihre Gesetze fest. Er lehrte die Gesetze von der Erhaltung des Stoffes und von der Erhaltung der Kraft, die Bruno schon genial erschaut hatte, wie er denn in ,,von der Ursache, dem Anfangsgrund und dem Einen“ sagt: „Das Universum, das ein großes Ebenbild und Abbild und die einheitliche Natur darstellt, ist ebenfalls alles, was sein kann, sofern die Arten und hauptsächlichsten Glieder in ihm dieselben bleiben und die Gesamtkraft der Materie sich als dieselbe erhält."

Auch für Gassendi ist die Bewegung die erste Ursache. Sie bewirkt die Verbindung und Trennung der Atome. Die Atome bilden die Substanz, die, nicht weiter teilbar, den Raum erfüllen und durch das Leere voneinander getrennt sind.

Dem Atomismus gegenüber erkannte Boyle nur eine, ausgedehnte, undurchdringliche, aber teilbare Substanz an. Die in ihr wohnende Kraft oder Bewegung zerlegte sie in kleinste Körperchen oder Korpuskeln von bestimmter Größe, Gestalt und Lage, die sich zu zusammengesetzten Körpern-Molekülen mischen können, so daß. die Korpuskeln den Elementen gleichkommen. Auch er führte alle Naturerscheinungen auf Bewegung, also auf Druck und StoB, auf Mechanik zurück, und alles Mechanische ist mathematisch bestimmbar.

Das System der. mathematischen Prinzipien der Naturphilosophie aufgestellt zu haben, ist das Verdienst von Newton.

Das Studium der Mathematik und der Natur bestimmen die Philosophie im 17. und dem folgenden Jahrhundert. Von der Mathematik nimmt der Rationalismus, von der Naturwissenschaft der Empirismus seinen Ausgang.

Unter den rationalistischen Denkern beschäftigte sich besonders Descartes mit dem Substanzproblem. Er ging vom methodologischen Zweifel aus, um die erste Grundwahrheit zu finden in dem berühmt gewordenen cogito sum. Die Tatsache des denkenden Bewußtseins 
war ihm der Beweis für die Wirklichkeit des denkenden Subjekts. Dieser Satz besaß, weil unmittelbar einleuchtend, Allgemeingültigkeit, er gehörte zu den eingeborenen Ideen. Darauf weiter aufbauend, erkannte er, daß alles gewiß ist, was wir ebenso klar und deutlich erkennen wie dieses cogito sum. Die höchste Idee, die wir in unscrem Geiste haben, ist die der Substanz. Er definiert die Substanz als das, was zu seiner Existenz keines anderen bedarf, also der ungeschaffene Gott. Die Gottesidee können wir nicht selbst durch Denkprozesse gebildet haben, da sie weit über alles hinausgeht, was auf Erden existiert. Sie kann uns daher nur von einem vollkommenen Wesen, von Gott selbst, eingegeben sein. $\mathrm{Zu}$ einem schlechthin vollkommenen Wesen gehört auch seine Existenz. Es ist derselbe ontologische Gottesbeweis, den schon Anselm aufstellte. Und schon damals wurde dagegen eingewandt, daß man die Realität eines Vorstellungsinhalts niemals wieder durch eine Vorstellung begründen könne. Anselms Gegner Gaunilo von Montigni aus dem Kloster Marmoutiers hielt ihm vor, daß die Vorstellung einer vorzüglichsten Insel durchaus kein Beweis sei für das Vorhandensein dieser vorziiglichsten Insel. Denselben Zirkelbeweis wie für das Dasein Gottes beging Descartes sogleich noch einmal, indem er von der Wahrhaftigkeit Gottes, der uns keine Scheinwelt vortrügen könne, auf die Wirklichkeit der Außenwelt schloß. Er unterschied neben Gott, der unendlichen Substanz, die Welt als die endliche Substanz. Diese geschaffene, endliche Substanz ist eine zweifache: Geist und Körper. Ihre konstitutiven Merkmale sind Denken und Ausdehnung. Geist und Denken, Körper und Ausdehnung sind dasselbe, sind identisch. Von dem Geiste haben wir, wie schon das cogito sum zeigt, ein unmittelbares Bewußtsein. Die Körper halten wir für wirklich, weil sie mathematisch bestimmbar sind. Die beiden endlichen Substanzen sind realiter von einander unterschieden, sie negieren sich, sie haben nichts miteinander gemein. Da aber das Denken doch im Körper, im Kopfe stattfindet, so mußte der Seele ein Sitz in diesem angewiesen werden. Der Platz mußte möglichst unräumlich, ohne Ausdehnung, punktförmig sein, und da die Zirbeldrüse der einzige unpaarige Teil des Gehirns ist, so wurde die Seele dort einquartiert. Zwar hatte Descartes eine gegenseitige Beeinflussung von Geist und Körper durchaus geleugnet, doch lehrte ihn der Augenschein, daß auf gewisse Denkprozesse stets gewisse körperliche Erschei- 
nungen folgen und umgekehrt. Diese Aufeinanderfolge erklärte or fïr Wirkungen der Lebensgeister, unendlich feiner Substanzen, die durch den ganzen Körper verteilt sind und zum Gehirn aufsteigen und hier den entsprechenden Vorgang auslösen. Nur durch die Lebensgeister kann die Seele den Leib zu Richtungsänderungen der Bewegungen veranlassen. Das Merkwürdige an der Descartesschen Substanzauffassung ist der doppelte Dualismus zwischen unendlicher und endlicher Substanz einerseits und der denkenden und ausgedehnten Substanz andrerseits. Geradezu aber eine Schwäche ist die Erfindung der Lebensgeister, um gewisse Wirkungen, die er erst negiert hatte, nun doch erklären zu können. An diesen beiden Punkten setzten seine Nachfolger ein.

Die Okkasionalisten erklärten sich gegen die Wechselwirkung der denkenden und ausgedehnten Substanz. Denn man kann nur das vollbringen, wovon man weiß, wie es geschieht. Wir wissen aber nicht, wie die Seele die Glieder zu Bewegungen veranlaßt. Sie schoben daher diese Veranlassung auf die unendliche Substanz, auf Gott ab und sagten: „Bei Gelegenheit" meines Willens bewegt Gott meinen Körper, bei „Gelegenheit" meiner Bewegung ruft Gott eine Vorstellung in meinem Geiste hervor.

Den Dualismus und die Wechselwirkungstheorie korrigierte Spinoza. Auch er definierte die Substanz als das, was Ursache seiner selbst ist: causa sui, ewig und unendlich. Mehrere Substanzen, mehrere Unendliche kann es nicht geben, denn sie könnten durch nichts voneinander unterschieden werden, sie wären identisch. Die Substanz oder Gott offenbart sich uns unter Attributen, deren Zahl unendlich ist, von denen wir aber nur zwei kennen, nämlich Denken und Ausdehnung, Geist und Körper. Diese sind keine Substanzen, weder als Ausfluß der göttlichen, noch als geschaffene oder untergeordnete, sondern sie sind der Ausdruck der Wesenheit Gottes. Die Attribute haben nichts miteinander gemeinsam, sie stehen in keiner Wechselwirkung zueinander, sondern ihre Äußerungen gehen nebeneinander her, sie entsprechen einander restlos. Zwischen Geist und Körper findet Parallelismus statt. Neben jedem körperlichen Vorgang läuft ein entsprechender geistiger her. Die Attribute Gottes stellen sich wieder in den Einzelobjekten dar, die modi oder Akzidenzien genannt werden. Auch der Mensch ist nur ein solcher modus, ohne selbständiges Dasein, nur an der und durch die Substanz existierend. 
Wie Descartes und Spinoza, so kam auch Leibniz von der Mathematik her an das Substanzproblem. Er wollte die Prinzipien der Mechanik fest begründen und fand, daß die Annahme einer ausgedehnten Masse nicht hinreichte, sondern daß noch der Begriff der Kraft hinzugenommen werden müßte. Er ging nun bei seiner Substanzerklärung von der Tatsache des Zusammengesetzten in der Welt aus und sagte: „Es muß eine einfache Substanz geben, weil es zusammengesetzte gibt." Denn das Zusammengesetzte ist nichts Anderes als eine Anhäufung des Einfachen. Leibniz schließt hier falsch. Denn die logische Notwendigkeit der Begriffsfolge ergibt noch keineswegs die reale Folge der Existenz der Dinge, die unter diesen Begriff fallen. Er schließt aus der Realität des Grundes des Zusammengesetzten auf die Wirklichkeit der logischen Folge des Einfachen. Das Zusammengesetzte ist teilbar, teilbar bis in das Unendliche, und auch bis in das Unendliche geteilt. Es liegt kein Grund vor, mit der Teilung bei sogenannten kleinsten Körpern oder Atomen halt $\mathrm{zu}$ machen, da ein Materielles immer geteilt werden kann, wenn es auch noch so klein ist. Das Einfache aber muß unteilbar sein. Er ging nicht wie Boyle auf eine chemische Lösung des Problems aus, für. den das Element der einfache unteilbare Stoff war. Er fand keine andere Lösung, als eine metaphysische. Nur das Geistige gibt uns unteilbare reale Einheit. Er setzt also die Körper aus einfachen unkörperlichen Bestandteilen zusammen und nannte diese Einheiten Monaden. Sie sind die wahre Substanz. Da die Monade unräumlich ist, so kann sie nicht von außen beeinflußt werden, sie entwickelt ihre eigenen Zustände aus sich heraus. Die Monade ist nicht das, was aus sich selbst ,ist", sondern das, was aus sich selbst „handelt", der Monade eignet das Moment der tätigen Kraft, die keine Anregung von außen braucht, wie es die Ansicht früherer Jahrhunderte war, sondern die sich selbst in Bewegung setzt. Die Monaden sind durch ihre Tätigkeit verschieden, alle einzelnen Dinge unterscheiden sich, selbst wenn sie scheinbar gleich sind, innerlich. Darum ist jede Monade ein cinzelner Körper nach dem Prinzip der Individualität. Denn wenn sie innerlich nicht unterscheidbar wären, dann wären sie in Wirklichkeit „ein“ Ding, sie wären absolut identisch nach dem principium identitatis indiscernibilium. Hieraus ergibt sich noch ein Weiteres. Jede Monade trägt die Gesetze ihrer Zustände in sich und zwar von Ewigkeit her, die Leibesmonaden ebenso wie 
die Seelemmomaden. Beide sind individuell, also ganz verschieden greartet, sie stehen demnach nicht in Wechselwirkung miteinander, sondern ihre Zustände laufen parallel und stimmen genau zueinander. 1)a die 'lätigkeiten aller Monaden genau zueinander passen, einander 'ntsprechen, so ergibt sich im Weltgeschehen eine Harmonie, die, weil von Ewigkeit her vorausbestimmt, die praestabilierte Harmonie heißt.

Während die rationalistischen Denker sich bemühten, der Substanz Realität zu verleihen, so suchten die Empiristen den Glauben an ihr Dascin zu erschüttern.

Locke war der erste, der den Substanzbegriff gehörig revidierte. Auf dem Wege der Analyse fand er, daß von den Körpern schlechterdings nichts uibrig bleibt, wenn man ihre einzelnen Eigenschaften abzieht, daß also ein Substrat, an oder in dem die Eigenschaften vorkommen, nicht vorhanden ist. Ebensowenig bilden die Eigenschaften eines Körpers eine durch Mischung entstandene, einheitliche Masse. Die Substanz ist daher weder der Träger noch die Summe der Eigenschaften eines Körpers. Es gibt keine Substanz, und doch kommen wir um die Idee der Substanz nicht herum, sie ist einmal da und läßt sich nicht wegleugnen. Sie ist das große $X$, das ,I know not what", sie ist ein Begriff, den zu bilden, unser Geist das .Vermögen hat.

Einen Schritt weiter ging Berkeley. Für ihn gibt es keinen Unterschied zwischen sekundären und primären Qualitäten, den Locke noch anerkannt hatte. Die ursprünglichen Eigenschaften: Größe, Gestalt, Lage, Bewegung existieren ebensowenig außerhalb unserer Vorstellung wie Farbe und Geruch. Es existiert nur unsere Idee von den Dingen, es gibt nur unsere Seele und das, was wir vorstellen. Nur Geister und deren Vorstellungen haben Realität, sie stammen von Gott und sind die eigentlichen Realia, die wahre Substanz. Alles andere löst sich in Schein auf. Demgemäß faßt er seine Substanzerklärung in die Worte zusammen: „,esse est percipi".

Berkeley erkannte die geistige Substanz noch an, für Hume fällt auch diese dahin. Ebensowenig wie dem Körper ein Substrat zugrunde liegt, ebensowenig liegt dem Geist oder der Seele ein beharrliches Selbst oder Ich zugrunde. Wie der Körper nichts ist als die Summe seiner Eigenschaften, so ist der Geist nur die Summe seiner inneren Zustände. Die Erscheinung einer körperlichen oder 
geistigen Substanz beruht nur auf dem Eindruck einer gleichmäßig wiederkehrenden Ideenassoziation. Die Ideenverbindungen sind jedoch zufällig und können sich ändern. Hume löst also die Substanz in Ideenassoziation auf.

Für die Empiristen ist die Substanz lediglich eine Form des Denkens, sie hat nur logische Bedeutung. Nur Berkeley erkannte cine Substanz an und zwar die des Geistes.

$\mathrm{Zu}$ einem ganz entgegengesetzten Resultat gelangten Hobbes und die französischen Materialisten, obgleich sie ihren Ausgangspunkt auch von der Empirie -nahmen. Sie leugneten die geistige Substanz. Denn jede Geistestätigkeit beruht auf innerer Bewegung, die durch eine äußere Bewegung hervorgerufen wird. Wir können uns äußere Bewegung njcht vorstellen ohne ein ihr zugrunde liegendes Substrat. Das zwingt uns, eine Materie oder Substanz anzunehmen. Diese Substanz besteht aus Atomen, besser Molekülen, die qualitativ voneinander verschieden sind und in denen die Bewegung als Selbsterhaltungstrieb liegt. Die Bewegung beruht auf der chemischen Wahlverwandtschaft, auf den Kräften der Anziehung und $\mathrm{Ab}$ stoßung.

Während Rationalisten und Empiristen ihren Ausgang von dem Erkenntnismittel, sei es die Vernunft, seien es die Sinne, nahmen, war die Grundfrage für Kant nicht: Welches ist das wahre Erkenntnismittel?, sondern die Frage: Wie kommt Erkenntnis zustande? So untersucht denn Kant auch in seiner transzendentalen Logik, wie sich die reinen Denkbegriffe zu den reinen Erfahrungen der Naturwissenschaften verhalten. 'Wie es keinen Inhalt ohne Form gibt, so muß auch jede Anschauung, die Erfahrung werden will, in die Kategorie eingegangen sein. Erst dadurch kommt eine Erkenntnis zu Wege. Umgekehrt dürfen wir auch die Kategorie nicht gebrauchen, wenn ihr nicht eine Anschauung zugrunde liegt. Die Kategorien sind Elementarbegriffe, Stammbegriffe des Verstandes, die das Mannigfaltige der sinnlichen Erscheinungen auf eine Einheit beziehen und zusammenfassen. Es gibt soviel Kategorien als es Verknüpfungs- weisen des Verstandes gibt, also 12 . Unter diesen 12 Kategorien befindet sich auch die der Substanz. Kant erklärt sie für das Konstante, sich selbst Gleichbleibende im Gegensatz zu den Zuständen, die die Dinge durchlaufen. Sie ist der Träger der Bewußtseinsvorgänge. Das Quantum der Substanzen kann weder vermehrt noch 
vernindert werden. Sie sind, sofern sie im Raume als zugleich wahrgenommen werden können, in durchgängiger Wechselwirkung. Die Substanz kann nur auf die Objekte der sinnlichen Erfahrung bezogen werden, sie gilt aber nicht für die intelligiblen Dinge ,an sich". Ob Kant selbst die intelligiblen Dinge ,an sich" leugnete, ist nicht sicher festzustellen. Gewiß weiß man, daß er ihre Erkennbarkeit leugnete. Demnach hat für Kant die Substanz weder eine ontologische noch eine logische Bedeutung, für ihn ist sie eine Form des Denkens und darum auch des Seins, nämlich des Seins in der Erfahrung; sie hat also erkenntnistheoretische Bedeutung.

Es schien, als hätte Kant mit seinem Kritizismus der Metaphysik für immer ein Ende bereitet. Aber das Bedürfnis des Menschen nach einer Erklärung der übersinnlichen Welt ist zu stark, als dab. es sich länger als auf kurze Zeit eindämmen ließe. Und so kommt um 1800 die große Reaktion gegen Kant, die an seinen Begriff des Dinges ,an sich" anknüpft. Er hatte verboten, die spekulative Vernunft auf die Dinge ,an sich“ anzuwenden. Es fehlte daher eine letzte Ableitung des Seienden aus einem obersten, höchsten, metaphysischen Prinzip, um das Kantische System einheitlich zu gestalten. Das Ende des 18. Jahrhunderts erwachte Spinozastudium führte zu diesem Prinzip hin. In Spinozas Identitätsgedanken war der Satz für die denknotwendige Ableitung gegeben, und so stellen die Systeme der großen Idealisten: Fichte; Schelling, Hegel, Schopenhauer eine Synthese von Kant und Spinoza dar.

Fichte negierte die Realität der Dinge ,an sich“, für ihn war der mundus intelligibilis dahingefallen. Man fragt sich, welche Stellung er ïberhaupt zu Sein und Denken einnahm, in welcher Synthesis er sie sich wiederfinden ließ. Da das Sein nichts mit dem Denken gemeinsam hat, so kann es dieses auch nicht hervorrufen. Wohl aber hat das Denken mit dem Sein ein gemeinsames Moment in dem Bewußtsein; denn es ist bewußtes = denkendes Sein. Also kann man aus dem Denken das Sein ableiten. Der erste gewisse Denksatz, der das Bewußtsein ausmacht, ist der Satz Spinozas von der Identität: $\mathrm{A}=\mathrm{A}$, der aber über die Existenz von A durchaus nichts sagt. Über die Existenz sagt der Identitätssatz erst etwas aus, wenn es heißt Ich $=$ Ich, das setzende Ich ist das gesetzte Ich. Das Ich setzt sich selbst, ist also causa sui, absolut. - Es ist causa sui wie die Substanz des Spinoza, doch aber fehlt ihm das Sein dieser Substanz als konstitu- 
tives Merkmal, es besitzt das Sein nur konsekutiv. Denn ursprüngliche Eigenschaft des Ich ist die Tätigkeit, und erst durch die Tätigkeit wird das Sein gesetzt, also ist dieses abgeleitet. Die Tätigkeit des. Ich ist auf sich selbst gerichtet. Das Ich selbst ist der 'Grund seiner eigenen Tätigkeit, und die Folge dieser Tätigkeit ist seine eigene Negierung. Es setzt damit das Nicht-Ich, nämlich die Welt des Seienden. Die Denktätigkeit hat die endlichen Gegenstände als Produkte. Da Ich und Nicht-Ich einander in demselben Ich negieren, so kämpfen sie miteinander um die Herrschaft, damit sie sich aber nicht vernichten, beschränkt sich ein jedes. Aus der beiderseitigen Beschränkung entsteht die Reflexion auf die Relationskategorien, aus ihrem gegenseitigen Kampfe entsteht die Produktion der Vorstellungsvermögen. Das Ich ist ein Ich der absoluten Position, das Gute schlechthin, das Nicht-Ich seine Negierung, die Welt, das Schlechte. Im Bewußtsein seiner selbst hat das Ich gegen das Nichtich anzukämpfen, der Welt seinen Charakter aufzuprägen, der in ihm liegt, es hat seine Bestimmung zu erfüllen. „Damit wir sollen können, dazu ist die Welt da."

So haben wir hier das Merkwürdige, daß Fichte mit dem Ich ein Absolutes, eine causa sui setzt ohne Substanz - d. h. ohne Seinscharakter. Anfänglich war Gott für ihn identisch mit dem absoluten Ich. Erst später erhob er ihn über das Ich, über die reine Tätigkeit, indem er ihm das reine Sein, also Substanz verlieh.

Schelling begann seine philosophische Laufbahn in geistiger Abhängigkeit von Fichte mit dem Gegensatz von Ich und NichtIch. Das Ich oder das Dènken ist ihm der Inbegriff des Subjektiven, das Nicht-Ich oder die Natur der Inbegriff des Objektiven. Ursprünglich sind Subjekt und Objekt identisch gewesen. Die Trennung fand erst statt, als die Intelligenz in uns bewußt ward und sich von der bewußtlosen Natur schied. Über Subjekt und Objekt erhebt sich als Einheit das Absolute, das alle Gegensätze in sich vereinigt, zur totalen Indifferenz, das Absolute, das identisch ist mit Subjekt und Objekt. Schelling denkt sich das Absolute als Substanz, als Gottheit ebenso wie Spinoza, nur daß er das Moment der Intelligenz stärker betont, das Absolute ist ihm absolute Vernunft, während Spinoza mehr die Ausdehnung hervorhebt. Und noch mit einem anderen Gedanken steht er in Gegensatz zu Spinoza. Bei ihm entwickelt die absolute Identität aus sich heraus die ganze Natur, von 
der anorganischen an bis hinauf zur organischen, bis hinauf zum Menschengehirn. Sp.noza aber dachte die absolute Substanz als immanente, die Natur ist ihm der Ausdruck des vollkomrnensten Wesens, aber sie hat sich nicht aus diesem allmählich entwickelt.

Auch Hegel bekennt sich zur Identitätsphilosophie des Spinoza. Das Absolute stellt auch ihm die Einheit von Gott und Natur dar. Zugleich aber macht sich der Einfluß seiner klassischen Studien geltend. Die Phiolosophie der Eleaten mit ihrer Behauptung: „Dasselbe ist Denken und Sein", und Heraklit mit seiner Lehre vom ewigen Werden waren bestmmend für seine Erklärung des Absoluten. Das Absolute, die wahre Wirklichkeit, ist der Gedanke, die Idee. Das Denken ist auch das Sein, beide sind identisch. Oberster allgemeinster Begriff ist das Sein. Nach der Methode der Selbstbewegung der Idee, die Thesis, Antithesis und Synthesis aufstellt, um die Wahrheit zu finden, sucht er das Gegenteil vom Sein und findet das Nichts. Sein und Nichts sind dasselbe. Denn sie sind beide qualitätslos. Bei dem Übergang vom Sein zum Nichts und vom Nichts zum Sein ensteht das Werden. In ihm treffen sich beide zur Einheit, das Werden ist ihre Wahrheit. So ist das Absolute bei Hegel-nicht die ruhende Substanz, sondern das sich selbst entwickelnde, lebendige Substanzsubjekt. Wie bei Plato existierte die Ideenwelt ursprünglich als ein System von Begriffen. Die Ideen modifizierten sich später in der unbewußten Natur, um dann im Menschen als Selbstbewußtsein zu erwachen. Im menschlichen Geiste erlangte die Idee ihre höchste Vollendung in den Schöpfungen der Kunst, der Religion und der Wissenschaft. Die Idee hat ihre Bestimmung erreicht und kehrt in sich selbst zurück. Jeder einzelne Gegenstand auf Erden stellt eine Entwickelungsstufe des Denkens, der Vernunft dar, und darum ist das Wirkliche das Vernünftige und das Vernünftige das Wirkliche. Hegel führt sein System konsequent durch.

Im Gegensatz dazu ist Schopenhauer inkonsequent genug. Er geht aus von den Kantischen Kategorien; die er auf eine einzige zurückführt, nämlich auf den Saizz vom zureichenden Grunde oder die Kategorie der Kauisalität. Dieser Satz hat eine 4 fache Wurzel, den Grund des Seins, des Geschehens, des Handelns und des Erkennens. Für uns kommt die 2. Wurzel in Betracht. Der Satz vom Grund des Geschehens fordert für jede Veränderung eine vorhergehende, die ihre Ursache ist, und eine Substanz als unveränderliches Substrat 
derselben, die Materie. Alle Veränderungen sind notwendig, alles Wirkliche ist materiell. Aber die Kategorie der Kausalität gilt nur für die Erscheinungen, nicht für die Gedankendinge und nur für die Zustände der Substanzen, nicht für diese selbst. Das wahre Wesen der Dinge „an sich" können wir nicht erkennen. Raum, Zeit und Kausalität trüben den Blick für die außer uns gegebene Welt. Ich sehe die Außenwelt nicht, wie sie ist, sondern wie meine Sinne sie malen. Nur ein Ding auf der Erde ist uns von außen und innen gegeben, das sind wir selbst, unser Selbstbewußtsein. Wir erblicken unseren Körper, und sofort fühlen wir uns, wissen wir uns als wollend. Unser Wille ist keine Vorstellung, sondern er ist das, was unser wahres Wesen ausmacht, das wahrhaft Seiende. Auf den Willensakt folgt augenblicklich die Körperbewegung, beide sind identisch. Der Wille ist nur der von innen gesehene Leib, der Körper der von außen gesehene Wille. Diese Erkenntnis überträgt Schopenhauer auf die ganze übrige Welt Wie unser Körper, so ist auch das Universum realisierter Wille. So haben z. B. die Steine den Willen zum Fallen. So erhebt sich hinter der Welt als Vorstellung die Welt als Wille. Das ,an sich" des Urwillens können wir nicht erkennen. Der Wille ist das wahrhaft Seiende, das Absolute, er ist ewig, ungeteilt, an sich unbewußt, einheitlich, er ist das Ein und Alles $\dot{\varepsilon} v x \alpha \iota \pi \alpha v$. Sein Wresen ist das Verlangen nach Realität. Er stellt sich materiell in immer vollkommeneren Stufen dar, in dem menschlichen Gehirn hat er sich ein Organ geschaffen, mit dem er sich selbst denkt. Gehirn und Denken sind dasselbe. Schopenhauer scheut sich nicht, seiner anfänglichen Behauptung, die Welt ist meine Vorstellung, die Materie ist nur Vorstellung, am Schluß den Satz gegenuiber zu stellen: „Die Vorstellung ist eine Gehirnfunktion."

Schopenhauer ist der letzte der großen Gelehrten, die im Anschluß an Kants Idealismus ihre Philosophie entwickeln. Je nach dem Punkt, den ihre Systeme besonders betonen, nennt man den Idealismus Fichtes den ethischen, den Schellings den physischen, den Hegels den logischen und den Schopenhauers den subjektiven. .Nach Abschluß dieser 4 großen idealistischen Systeme treten die Geisteswissenschaften ihr Zepter an die mathematischen Naturwissenschaften ab. Die Philosophie der neueren Zeit ist von diesen beherrscht.

Herbart geht von der Erfahrung aus an den Substanzbegriff 
heran. Die Erfahrung lehrt den Gegensatz von Erscheinung und Scin, sie lehrt, daß jeder Erscheinung ein Scin zugrunde liegt. Was dic Dinge an sich wirklich sind, können wir nicht erkennen, weil sie uns nicht unmittelbar gegeben sind. Wir können nur aus der Erscheinung auf das Sein schließen. Da die Dinge der Außenwelt viele Eigenschaften haben, so müssen wir aus der Vielheit der Erscheinungen auf die Vielheit des Seins schließen. Weil die Gegenstände der Erscheinungswelt die Summe vieler Eigenschaften sind, so ist auch das Ding ,an sich", das wahre Sein, ein Zusammen vieler, einfacher Realen. Jedes Reale oder jede Monade ist uranfänglich, unveränderlich, voneinander verschieden, raumlos und ohne innere Zustände. Er bezeichnet seinen Standpunkt als den des qualitativen Atomismus. Obgleich er mit Leibnitz den Begriff der übersinnlichen Monade gemeinsam hat, so folgt er ihm doch nicht in seiner Lehre von der praestabilierten Harmonie. Jedes Reale hat Individualität, aber ihre Zustände sind nicht aufeinander abgestimmt, sondern sie widersprechen, sie stören, sie hemmen einander. Darum ist das Wesen der Realen der Trieb der Selbsterhaltung. Dieses Verlangen nach Selbsterhaltung ist das einzige Geschehen, die einzige Veränderung, auf sie ist die Welt der Erscheinung zurückzuführen. Nähẹr als alle anderen Realen liegt uns unsere Seele, die sich im Gehirn befindet und, weil sie als Reales raumlos ist, punktförmig gedacht wird. Auch sie übt Selbsterhaltung gegen die Störungen, gegen die Vorstellungen. Herbart faßt die Vorstellungen als Kräfte auf, Kräfte kann man messen und berechnen, und so macht er den Versuch, Statik und Mechanik auf die Seelenkräfte, die Vorstellungen, anzuwenden, ein Versuch, der ihm mißlang.

Fechner war es, der an diesen Gedanken Herbarts wieder anknüpfte. Das Naturgesetz von der Erhaltung der Energie sollte ihm den Weg weisen. Er glaubte, daß jedem geistigen Vorgange ein körperlicher zugrunde liege. Beide stehen in einem bestimmten Verhältnis zueinander, etwa wie innere und äußere Seite des Kreises. Bestimmte Verhältnisse kann man messen. Man mußte nur die Maßeinheit der geistigen und körperlichen Vorgänge, der Vorstellungen und ihrer körperlichen Ursache finden, und diese lieferte das Gesetz von der Erhaltung der Energie, das für beide Arten von Vorgängen gilt. Die psychische Intensität wächst nicht so schnell wie der entsprechende physische Eindruck, sondern nur im Verhältnis des Reiz- 
zuwachses zu der schon vorhandenen Reizstärke. Auf Grund von Beobachtungen war dieses Verhältnis festzustellen, was auch gelang. Das Experiment war damit in die Seelenlehre eingeführt. Zugleich war die Auffindung der psychisch-physischen Formel der Beweis, daß tatsächlich ein Parallelismus der geistigen und körperlichen Vorgänge stattfindet. Fechner schloß aus dem Parallelismus im Menschen auf den Parallelismus im Universum. Überall entsprechen Geistiges und Körperliches einander. Unendliches und Endliches, Gott und Welt, bilden eine Einheit wie Seele und Körper im Menschen. Beide, Unendliches und Endliches, bauen sich auf einer Einheit auf, die höchste geistige Einheit ist das Gottesbewußtsein, die letzte körperliche das Atom. Er faßt das Atom allerdings nur als wissenschaftliches Hilfsmittel auf, um kleinste Kraftzentren zu gewinnen. Da jedem Körper ein Geistiges zugrunde liegt, so hat alles Endliche auch Seele, Organismen wie Anorganisches. Alles ist beseelt. Die Einheit dieser einzelnen Seelen ist die göttliche, die Weltseele, mit der sie nach dem Tode einen, von räumlich-körperlichen Schranken befreiten Zusammenhang bilden. Er stellt diese Lehre von den Seelen als „Tagansicht" der atomistischen „Nachtansicht" gegenüber.

Auch Fechners Schüler Lotze unterscheidet zwischen der äußeren und inneren, der endlichen und der unendlichen Welt. In der endlichen Welt, in der Welt des Scheins, herrscht die Notwendigkeit, die Mechanik. Die Welt des Scheins setzt sich aus Atomen zusammen, die im Kausal- und Wechselwirkungsverhältnis zueinander stehen. Hinter dieser endlichen Welt steht die unendliche, die Welt des Seins, die sich aus geistigen Realitäten zusammensetzt. Wie die Gesamtheit der Atome die endliche Materie ausmachen, so bilden die Seelen, die geistigen Realitäten, die Ursubstanz. Diese ist absolut, aber nicht qualitätslos, sondern absolute Persönlichkeit. Ihr Gesetz ist nicht die Notwendigkeit, der Mechanik der endlichen Welt entspricht der Zweck der unendlichen. Der Zweck der Ursubstanz ist die Realisierung der Welt der Werte. Jedes Reale verfolgt einen Zweck, trägt eine Idee in sich, die es verwirklichen soll, und daher stehen alle Realen - in Beziehung, in Wechselwirkung zueinander. "Sein" heißt also bei Lotze in Wechselwirkung stehen, und „das Sein" ist die Ursub$\operatorname{stanz}$, die absolüte Persönlichkeit.

Wie Lotze und Fechner, so wandte auch von Hartmann die naturwissenschaftliche Methode für seine Erklärung der Substanz 
an. Die Dinge „an sich" haben eine reale Existenz außerhalb unserer Vorstellung. Das Wesen der Ideenwelt ist Tätigkeit, ist mit sich sclbst identische Wirksamkeit, es ist die unbewuBte Einheit von Vorstellung und Wille. Von Hartmann erhebt zur obersten Einheit das Unbewußte. Das Unbewußte ist die Substanz, es produziert den Bewußtscinsinhalt und die Materie, also geistige und körperliche Welt.

Von Hartmann war es, der in seiner Kategorienlehre wieder die Frage aufwarf, ob der Substanzbegriff nur eine Form unseres Denkens oder, weil eine Form unseres Denkens, auch die des Seins ,an sich" sei.

Die Beschäftigung mit dem Substanzproblem zeigt, daß es ein metaphysisches Problem sei, denn es führt über die Erfahrung hinaus in das Außersinnliche. Das Übersinnliche ist uns vernunftmäßig gegeben. Daher war die Metaphysik immer mit der Methode der Dedulation verbunden. Erst durch den Einfluß der Naturwissenschaften verfielen Lotze, Fechner und Hartmann darauf, die Induktion und das Experiment auf die Metaphysik anzuwenden und so der Metaphysik von „oben“ die von „unten“ entgegenzusetzen. Das einzige außersinnliche Sein, das uns gegeben ist, ist unser eigenes Denken, unsere Seele. Deshalb unternahmen es jene Philosophen, die Seele naturwissenschaftlich zu betrachten, ihre Zustände, ihre Wirkungen zu beobachten, ihre Gesetze mathematisch zu bestimmen. Indem die moderne Psychologie die Seele, das geistige Wesen studiert, hofft sie dem Übersinnlichen näher zu kommen, ebenso wie die Naturwissenschaft die sinnliche Materie ergründen will, indem sie den Begriff des Körpers feststellt. Beide Wissenschaften arbeiten an ihrem Teil zur Klärung des Substanzproblems mit. Sie stellen die Grundtatsachen, die Grundbegriffe fest.

Für die moderne Naturwissenschaft ist der Körper das Element, aus dem die Substanz sich zusammensetzt. Die Außenwelt besteht aus einem Inbegriff von Körpern, die in Raum und Zeit gegeben sind. Der Körper ist relativ beharrlich, im Gegensatz zur früheren Ansicht, nach der die Substanz absolut beharrlich war. Der Körper ist ferner im Raum und er muß einen Raum erfüllen. Die Größe der Raumerfüllung ist bestimmt durch den Begriff des Raumes, der ihn dreidimensional gibt. Demnach hat auch der Körper drei Dimensionen, seine Gestalt verändert sich nicht, wenn er sich im Raume 
verschiebt. Ein Körper ist ein einzelner Körper nur durch seine zeitlich-räumlichen Bestimmungen, nicht aber durch ein principe interne. Denn zeitlich kann an demselben Orte nicht noch ein zweiter Gegenstand sein. Der Körper ist auch eine Masseneinheit, er hat Volumen, ist undurchdringlich. Die Masse des Körpers ist das, was bei allem Wechsel beständig bleibt, was bei aller Veränderlichkeit feststeht. Die Masse ist der Realgrund für die Undurchdringlichkeit. Da die Masse das Konstante ist, so galt es vor allen Dingen, sie zahlenund größenmäßig genau zu erfassen. Da zeigte die Forschung, daß nur eine Erfahungstatsache an der Masse gegeben ist.: Körper erfahren beschleunigte Bewegung. Die Auffindung der Fallgesetze zeigte dann weiter, daß die Masse der Realgrund der Trägheit, des Beharrungsvermögens der Körper ist, die Masse, die man hier als Schwerkraft auffaßte. In der Schwere hatte man die Grundkraft der Materie entdeckt. Und so löste die mechanische Naturauffassung die Körperwelt in die Bewegungen der Atome auf. Im Gegensatz dazu fand Ostwald die Einheit von Materie und Geist in der Energie. Alles ist Arbeit, mechanische, magnetische, elektrische, chemische Energie. Wieder andere halten die Elektronen für die Elemente des Seins, der Materie und hoffen, von hier aus eine neue, die wahre Substanzerklärung zu finden.

So stellt die Geschichte der Philosophie „das Ringen um die Kategorie der Substanz" dar. Wir wollen diese Erkenntnis erweitern und sagen, das Ringen um die Erkenntnis der Substanz ist die Geschichte der Menschheit überhaupt, und die Stellung des Menschen zu diesem Problem ist sein Schicksal. Und doch müssen wir uns bescheiden. Wir können der Lösung nur immer näher kommen. Es ist dem Menschen gesetzt, die Wahrheit zu suchen, das Finden ist die Vollendung. Und so bleibt das. Endliche für die Forschung, aber das Unendliche, das wir im Geiste erschauen, das beten wir an. 